\title{
Secondary Hemochromatosis due to Alcoholic Liver Disease
}

\author{
Koichiro Yamamoto ${ }^{1}$, Yoshito Nishimura ${ }^{1}$, Mai Yamane ${ }^{1}$, Reina Son ${ }^{1}$, Daisuke Omura ${ }^{1}$, \\ Kou Hasegawa ${ }^{2}$, Youhei Marukawa ${ }^{2}$, and Fumio Otsuka ${ }^{3}$ \\ ${ }^{1}$ Okayama University Hospital \\ ${ }^{2}$ Okayama University Graduate School of Medicine Dentistry and Pharmaceutical Sciences \\ ${ }^{3}$ Okayama Daigaku - Shikata Campus
}

June 12, 2020

\section{Key Clinical Message:}

Clinicians should recognize the importance of evaluating iron overload in those with alcoholic liver diseases. Markedly elevated serum ferritin levels are a diagnostic clue for secondary hemochromatosis, necessitating the iron depletion therapy.

\section{Case:}

A 50-year-old man with alcoholism was admitted to our hospital with a week-long history of anorexia and vomiting. Physical examination revealed jaundice. Laboratory findings showed increased hepatobiliary enzymes (total bilirubin $5.42 \mathrm{mg} / \mathrm{dL}$, aspartate aminotransferase $360 \mathrm{U} / \mathrm{L}$, alanine aminotransferase $166 \mathrm{U} / \mathrm{L}$ ). Further evaluation revealed hyperferritinemia $(5,988 \mathrm{ng} / \mathrm{mL})$ and elevated transferrin saturation (96\%). Magnetic resonance imaging revealed that liver and pancreas had low signal intensity on the T2-weighted image (Figure 1A ) and lower signal intensity on the gradient-echo in-phase image than the out-of-phase image (Figure 1B, 1C ). Based on these findings, acute alcoholic liver disease with secondary hemochromatosis was diagnosed. Complete bed rest with alcohol cessation improved his symptoms. He was discharged with an alcoholic rehabilitation program appointment.

Hemochromatosis is a systemic disorder with excessive total body iron storages and deposition ${ }^{1}$. Secondary hemochromatosis occurs with iron overload that is not attributable to primary abnormalities in iron metabolism. Although excessive alcohol consumption induce iron metabolism disturbance, serum ferritin level over $1,000 \mathrm{ng} / \mathrm{mL}$ is considered rare $^{2}$, which mandates close follow-up. As excessive iron overload could be treated with iron depletion therapy, clinicians should realize the importance of evaluating the extent of iron overload in patients with known liver diseases, including alcoholic liver disease.

Acknowledgments: None.

Conflict of Interest: The authors declare no conflicts of interest.

Authors' Contribution:

$\mathrm{KY}$ and $\mathrm{YN}$ : wrote the first draft and managed all the submission process. KH and FO: contributed to the clinical management of the patients and revised the manuscript.

\section{References}

1. Gattermann N. The treatment of secondary hemochromatosis. Dtsch Arztebl Int. 2009;106(30):499-504, I. 
2. Bell H, Skinningsrud A, Raknerud N, Try K. Serum ferritin and transferrin saturation in patients with chronic alcoholic and non-alcoholic liver diseases. J Intern Med. 1994;236(3):315-322.

\section{Figure Legend}

Figure 1.

(A ) Liver magnetic resonance imaging shows the low signal intensity of the liver (arrows) and pancreas (arrowheads) on the T2-weighted image. (B, C ) The gradient-echo in-phase (B) and out-of-phase (C) sequences reveal the lower signal intensity of the liver on the in-phase image compared with that of the out-of-phase.

\section{Figure 1}

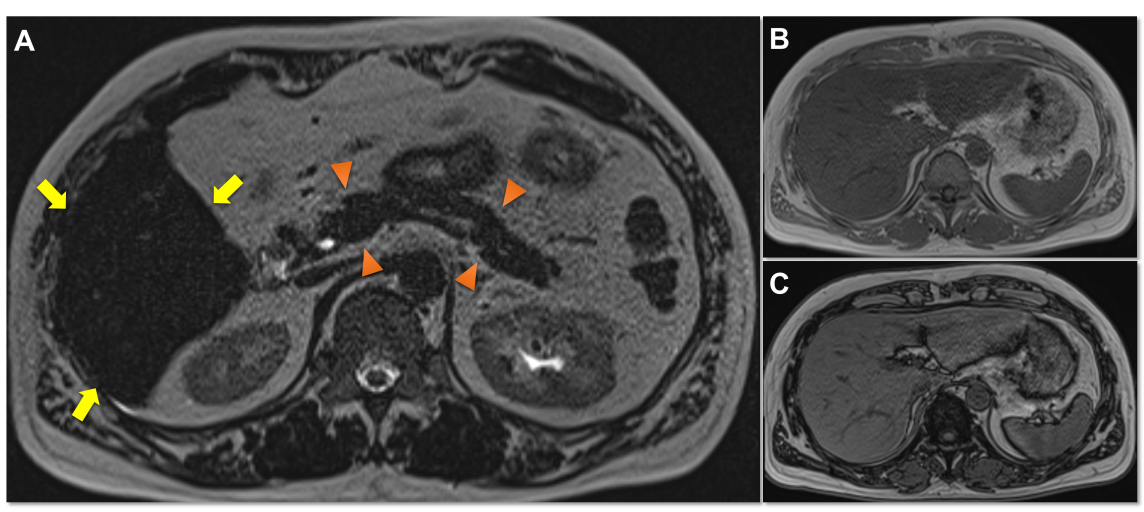

